\title{
Identification of non-technical skills from the resilience engineering perspective: a case study of an electricity distributor
}

\author{
Priscila Wachs ${ }^{\mathrm{a},}$, , Angela Weber Righi ${ }^{\mathrm{a}}$ and Tarcisio Abreu Saurin ${ }^{\mathrm{a}}$ \\ a Industrial Engineering and Transportation Department, Federal University of Rio Grande do Sul, Av. Oswaldo \\ Aranha 99- $5^{\text {th }}$ Floor, Porto Alegre, RS, Brazil
}

\begin{abstract}
Training in non-technical skills (NTS) does not usually question the design of the work system, and thus focuses narrowly on workers as the unit of analysis. This study discusses how the identification of NTS, a major step for developing an NTS training program, might be re-interpreted from the perspective of resilience engineering (RE). This discussion is based on a case study of identifying NTS for electricians who perform emergency maintenance activities in an electricity distribution power line. The results of the case study point out that four data analysis procedures might operationalize the RE perspective: (a) identifying factors that make the work difficult and which could be integrated into NTS training scenarios; (b) identifying recommendations for re-designing the system, in order either to reduce or eliminate the need for NTS; (c) classifying the NTS into pragmatic categories, which are meaningful for workers; and (d) regarding the identification of NTS as an opportunity to give visibility to adaptations carried out by workers.
\end{abstract}

Keywords: Non-technical skills, resilience engineering, safety at work, electricians

\section{Introduction}

A professional skill is a particular way to perform a task, which involves both technical skills (TS) and non-technical skills (NTS). Although it is not possible to differentiate both types strictly, a TS is defined as using the intellect, dexterity and motor coordination to deal with materials, tools and procedures [11]. NTS are personal, social and cognitive skills that complement TS, which may contribute to safe and efficient work [13].

NTS have been classified into broad categories, such as situation awareness, decision making and communication $[1,13]$. While these categories can be investigated from various theoretical perspectives, the studies that label themselves as studying NTS, usually adopt behaviorist paradigms and are based on information processing theory. This means that they focus on cognition in the mind, rather than on cognition in the wild (i.e., regarding cognition as a distributed phenomenon in the socio-technical system) [7,
16], and pays little attention to the context that produces behaviors.

This approach to investigating NTS has received a number of criticisms [2, 15], since: (a) neglecting the context makes it easier to blame the operators when the performance is not as expected, (b) it takes for granted that operators should use NTS as automated routines, rather than using them in a flexible manner, supported by designing the socio-technical system adequately; and (c) given that NTS are seen as procedures to be followed in a standardized way, they become less generalizable and less useful in unexpected situations. Moreover, organizations that have adopted safety management practices based on behaviorist epistemological assumptions, have reached a performance plateau [12].

Thus, innovative perspectives are necessary if NTS knowledge and practice are to progress. This study proposes to approach NTS from the perspective of resilience engineering (RE), which aims at contributing to the safety of complex systems, by measur-

${ }^{*}$ Corresponding author. E-mail: priscilawachs@producao.ufrgs.br 
ing and improving their resilience [3]. A major assumption of this study is that, from the RE perspective, the practice of NTS does not depend solely on the operators' skills. A supportive working environment is also necessary either to reduce or eliminate the need for NTS.

However, no guidelines have been identified in the literature on how to operationalize the RE perspective on NTS. In this study, what is investigated is how this operationalization might happen when NTS are being identified, a critical step for developing an NTS training program. Therefore, the main research question of this paper might be stated as follows: how are NTS to be identified from the RE perspective?

The research question is empirically investigated by examining the work of grid electricians. This sector was chosen due to the variety of working conditions that workers are exposed to, which tends to demand the use of NTS more intensively in comparison with more stable domains. Two additional factors justify the choice of this sector: no previous studies were found about what NTS are necessary for grid electricians; and, secondly, this study is part of a broader research project on RE applications in electricity distribution. Moreover, as part of this project, a previous study pointed out the need to improve the training practices of the company investigated [17] and therefore further investigation of this need seemed likely to inform how to address the research question of this paper.

\section{Research Method}

\subsection{The company investigated}

The company investigated distributes electricity to 72 cities in south of Brazil, a total of 1.44 million consumers. There are $50,400 \mathrm{~km}$ of power lines, 46,500 transformers and 830,000 poles. Its workforce consists of about 2,000 employees, as well as a variable contingent of outsourced electricity workers. The typical functions of an electricity distribution company are: (a) designing, constructing and renovating power lines; (b) undertaking preventive maintenance of the power line; (c) performing emergency maintenance to re-establish the power supply; (d) connecting new customers to the system; and (e) disconnecting customers from the system [8].

\subsection{The design of the research}

The study focused on teams who undertake emergency maintenance jobs on dead lines. According to reports by the company's representatives, these teams are the ones exposed to the worst working conditions.

The data collection and analysis procedures adopted the recommendations proposed by cognitive task analysis (CTA), since they are well-known for enabling informal working practices to be elicited. Also, a number of studies report successful applications of CTA as to identifying NTS [4, 10]. Thus, three main sources of data were used: interviews, observations and document analysis. The observations contributed indirectly to identifying NTS, as they were not systematically conducted.

It is worth emphasizing that the process of identifying the results of interest to this project was timeconsuming, due to the large volume of qualitative data produced by CTA, and required at least two researchers to be involved at each stage of the cycle.

\subsection{Interviews}

The Critical Decisions Method (CDM) was used to conduct the interviews, since it enables how experts dealt with challenging situations to be investigated, situations in which it is usual for tacit knowledge and NTS to make a difference.

The interviews were conducted with pairs of electricians, since this was the arrangement under which the emergency crews worked in the company. Each interview lasted nearly one hour and a half. In total, 13 interviews were conducted, resulting in approximately 20 hours of interviews and 52 hours of transcription. The interviews were recorded with the approval of the interviewees. The transcripts were analyzed, separately, by two researchers. For each transcript, the researcher identified sections of the text that were associated with the three objects of interest: NTS; factors that could be integrated within NTS training scenarios; and actions to re-design the system, which could either eliminate or reduce the need for NTS. Then, both researchers compared their lists with the three objects of interest (i.e., NTS, simulation factors, actions to re-design the system), and reached a consensus. The decision to highlight excerpts from interviews excerpts, rather than isolated words or expressions, contributed to overall understanding and successful retrieval from the database, of the context in which the outcomes of interest were obtained. 


\subsection{Document analysis}

The two main documents consulted to gather relevant data for the purposes of this study were accident investigation reports and incident reports. As to accident reports, those that occurred in the power generation or transmission, with population or consumers, or during transportation were excluded. Based on these criteria, 61 out of the 144 reports available were selected.

Similarly, 57 incident reports forms, filled out by emergency teams, were analyzed. In this incident reporting system, incidents were interpreted as any event or condition of lack of safety. The analysis of documents adopted procedures that were similar to those adopted for analyzing the interviews.

\subsection{Observations}

The main opportunity to carry out observations was during the period in which two members of the research team attended about 30 hours of the electricians' induction training course. An additional 20 hours of observations was undertaken as the researchers joined a team of electricians during field work. In addition to their role in the triangulation of data, observations helped to establish a minimum theoretical and practical technical knowledge base for the researchers, which was essential to support the analysis of the data collected.

\subsection{Framework for data analysis}

The NTS were identified and classified across two levels of abstraction. Initially, they were identified at the less abstract level (referred to as examples of NTS), and extracted directly from the transcriptions of the interviews and the accident and incident forms. The second level, referred to as NTS categories, is defined by labels, for each of which various examples are given.

The factors that could be integrated into the simulation scenarios were similarly organized. At the less abstract level, work constraints that could be incorporated into training scenarios (e.g., cables that are difficult to make out at night) were identified. At another level, these difficulties were grouped into categories (e.g., night), based on the similarity among the factors. The actions for re-designing the work system were organized at only one level of abstraction.

Also, both the factors to be integrated into simulation scenarios and recommendations for the re-design of the system were grouped according to their links to the sub-systems of a socio-technical system [6], namely: those of the social, technical, work organization and external environment.

\subsection{Validation of the results}

There were two formal moments for validating the results. The first was a meeting, lasting three hours, involving 24 company members (e.g., staff from the Human Resources Department, Training Department and electricians) and the research team. However, the main opportunities for validating the results were two meetings, each lasting four hours, involving two expert electricians and two researchers.

\section{Results and discussion}

\subsection{Main characteristics of the work of emergency maintenance teams}

In the company investigated, not including subcontractors, the total population of electricians who worked exclusively on emergency maintenance was 170 people, all male. The work has three main objectives, to which NTS can contribute: (a) performing the tasks safely, both for electricians and for the population; (b) minimizing the time customers are without power; and (c) minimizing the number of customers without power during maintenance. The last two are also corporate objectives, since the regulator evaluates the companies of this sector based on indicators related to these objectives. As to safety, despite there being a formal safety policy, an earlier study in this same company had identified evidence of a weak safety culture [17]. The management of trade-offs between the previously mentioned objectives is part of the normal work routine.

The demand for services of the emergency maintenance teams usually starts with a phone call from end customers to the Customer Service Call Center, which sends a service order, via a computerized system, to the operations center. Then, this center contacts the teams that are near the place where the call came from. Typically, customer demand is due to the shortage of power and, sometimes, due to damages to the distribution power line infrastructure.

All tasks in the field are undertaken in close collaboration with the operations center, which is in contact with the electricians by radio or telephone. The major functions of the operations center are as fol- 
lows: (a) to inform the teams where (i.e., which address) their support is necessary; (b) to request additional resources when necessary (e.g., to call additional teams); (c) to guide electricians about which parts of the power line are energized and which are not; to open and close keys remotely, blocking or releasing the passage of electric current in given stretches.

In operational terms, the electricians' work usually involves the transportation of materials, driving motor vehicles, working at ground level and working at various heights, on the poles. The work demand increases on rainy and windy days, and overtime is also frequent.

Indeed, the work environment is variable, since the distribution power line is widely dispersed in space, as it crosses both urban and rural areas, is exposed to different geographical characteristics and has to meet customers' socio-economic profiles, and so forth. Moreover, the variability in the distribution power line in itself is substantial. Although there is a formal design of the power line structure, it is shared with other companies (e.g., cable TV) and public organizations, which creates a need for coordination between the parties.

\subsection{NTS identified in the case study}

Based on the data collected, 105 examples and 12 categories of NTS were identified (Table 1). The premise on which the choice of the labels that designate the categories was based was that employees should find them meaningful and simple to understand. An excerpt from an interview illustrates how these results were obtained and how they can be tracked down for re-interpretation, if necessary: "what could be the cause of the defect in this case?
We looked for an illegal connection (i.e., a fraud made by the client) and we did not find any; we looked for a loose cable and we did not find one; we looked for a broken insulator causing energy to drain away and we did not find any. The cause of the defect was not easily visible, since the most obvious causes for defects in that context had already been checked"

The above statement gave rise to the following example of NTS: "initially, look for visible causes of power shortage, such as broken insulators, loose cables and fallen poles." In turn, this example was associated with NTS 7 (devising strategies to identify defects in the power line). In fact, the association of each example with only one category of NTS should be interpreted as a compromise solution, which took into account the readability and organization of a large volume of data. Indeed, the example of NTS previously mentioned could also be associated, without inconsistency, with NTS 5 (identifying nonstandard structures, materials or equipment).

Had either the well-known NTS categories proposed by Flin et al. [13] or the macrocognitive functions proposed by Klein [5] been adopted as labels, the overlaps among the categories would persist. Nevertheless, rather than pointing to a weakness of data analysis, this conclusion is a result of distributed cognition and the complexity of the work environment investigated. Thus, both for the purposes of data classification and communicating this to workers, the theoretical meaning of the labels is secondary in relation to their pragmatic role. Moreover, the decision not to adopt labels proposed in previous studies [13] avoids making unnecessary commitments to categories that have been heavily criticized due to their behaviorist theoretical assumptions [14].

Table 1

NTS categories, number of examples and number of interviews and documents excerpts

\begin{tabular}{|c|c|c|c|}
\hline NTS & Description & $\begin{array}{c}\text { No. of } \\
\text { examples }\end{array}$ & $\begin{array}{l}\text { No. of } \\
\text { stretches }\end{array}$ \\
\hline 1 & To discuss with the operation team defining procedures that should be taken and obtaining line information & 10 & 35 \\
\hline 2 & To discuss with the field team reaching a common understanding on the situation & 3 & 17 \\
\hline 3 & To discuss with the consumers and population the status and risks of line maintenance procedures & 4 & 7 \\
\hline 4 & To express doubts, fears and requests for help from other team members & 4 & 6 \\
\hline 5 & To identify structure, lines or equipment that are non-standard, damaged or have failed & 20 & 63 \\
\hline 6 & $\begin{array}{l}\text { To identify visible signs in the environment that indicate difficulties in doing the task or the probable cause } \\
\text { of damages to the line }\end{array}$ & 7 & 16 \\
\hline 7 & To develop strategies to identify line defects & 12 & 35 \\
\hline 8 & To develop work strategies, after the defects have been identified & 15 & 40 \\
\hline 9 & To plan and to check the equipment and materials that are necessary to undertake the task & 4 & 19 \\
\hline 10 & To distribute the tasks between team members and to do the task accordingly & 3 & 19 \\
\hline 11 & To identify causes of stress and fatigue & 12 & 29 \\
\hline \multirow[t]{2}{*}{12} & To develop strategies to cope with stress and fatigue situations & 11 & 19 \\
\hline & Totals & 105 & 305 \\
\hline
\end{tabular}




\subsection{NTS and adaptations of procedures}

Due to the RE perspective, there was a particular interest in finding the relationship between NTS and adaptations to procedures. Two types of relationship were detected: NTS examples that are adaptations of procedures and NTS that are needed because procedures have been adapted. One example of the former is the decision not to disconnect illegal extensions, but to reposition them. In the latter, what can be cited is the ability to identify an improvised connection to the electricity line, made due to lack of suitable materials, by a team that was previously in the same place.

Although the adaptation of procedures, whether or not associated with NTS, is given no formal recognition by higher hierarchical levels, such adaptations are common in many companies, in spite of the new hazards that they often introduced.

\subsection{Factors that can constitute training scenarios in NTS}

The factors that could constitute training scenarios in NTSs were organized into fourteen groups, each having a series of examples (Figure 1). Since the research project foresees the training scenarios being simulated ones, first and foremost, by physical means in the field, the technical feasibility of simulating each factor was analysed at the meetings with the experts in which the results were validated. Based on this meeting, it was seen that only three factors should be excluded, all of which were associated with the external environment: adverse weather conditions, region in turmoil because of urban violence and the presence of animals and insects. In fact, technical factors related to the subsystem can be simulated with relative ease, by modifying or removing equipment, materials and tools. As to the factors associated with the organization of work and social sub-systems, they can be simulated by means of the behaviors and characteristics of the individuals participating in the scenario.

All the factors for simulation are associated, to a lesser or greater degree of intensity, with all NTS categories listed in Table 1. Taking into consideration only the possible combinations among the eleven factors judged capable of simulation, the large number of options for the design of scenarios is noted. Unlike what happens with NTSs, which are present to a greater or lesser degree in all scenarios, the factors for simulation can be interpreted as variables subject to manipulation by the instructors, and may or may not be inserted into each training session.

Figure 1

Factors which can constitute training scenarios in NTS

\begin{tabular}{|l|}
\hline \multicolumn{1}{|c|}{ Factors associated with the technical sub-system } \\
\hline Activity carried out previously in an inadequate way (e.g., pole without identification, direct connection) \\
\hline $\begin{array}{l}\text { Lack of equipment or materials to undertake the activity (e.g., lack of pulleys to hoist materials, lack of collective or individual protective } \\
\text { equipment) }\end{array}$ \\
\hline Failure in power line equipment or materials (e.g. rotten pole, lightning arresters, fuse switch, sectionaliser) \\
\hline Lifting weights and need to use a lot of physical strength (e.g. wet tools, heavy tree branches, many ramifications in the power line) \\
\hline \multicolumn{1}{c|}{ Factors associated with the organization of work and social sub-systems } \\
\hline Long working hours (e.g. overtime, without intervals for meals) \\
\hline $\begin{array}{l}\text { Lack of support from colleague (e.g. relationship difficulties, an electrician who is on the ground is doing other tasks instead of monitoring } \\
\text { and supporting the colleague who is on the pole) }\end{array}$ \\
\hline Pressures from supervisors, operations center or users (for example, complaints about the delay in accomplishing the task) \\
\hline $\begin{array}{l}\text { Problems at the interface between the electricians and central operations (e.g. no radio signal, long waiting time before receiving orders } \\
\text { from the center, difficulty of relationship) }\end{array}$ \\
\hline \multicolumn{1}{c|}{ Factors associated with the external environment sub-system } \\
\hline Difficulty of access to the region (e.g. fences, long distances, undergrowth, branches, sand, swamps) \\
\hline Difficulty of access to the power line (e.g. vegetation, interference from street lighting, poles in consumers' yards, clandestine connections) \\
\hline Night (e.g. difficulty of visualizing the power line and equipment at night) \\
\hline Adverse weather conditions (e.g. rain, extreme cold or heat)] \\
\hline Region in turmoil because of urban violence (e.g. threats to the population so that illegal lines are not cut off) \\
\hline Presence of animals or insects (e.g. bees and dogs) \\
\hline
\end{tabular}


3.5. Actions of re-designing the socio-technical system which could reduce or eliminate the need to conduct NTSs

Figure 2 lists actions for the re-design of the sociotechnical system that could reduce or eliminate the need for conducting NTSs. The existence of actions associated with all the sub-systems of a sociotechnical system reinforces the need to use the RE perspective, as it is clear that the conduct of the NTSs depends on the design of the whole system, not just that of training the workers.

Examples of actions that may be cited are reducing illegal connections and expanding preventive maintenance in the power line. These two actions would contribute to reducing the need to use NTSs 5 and 6 (since there would be less need to identify nonstandard structures and physical obstacles to carrying out the tasks), NTS 11 (identifying sources of fatigue and stress) and NTS 12 (developing strategies for dealing with stress and fatigue), as a power line in a better state would reduce the physical wear and stress in performing the work.

It can also be seen that several actions are difficult to implement because they depend on corporate policies and decisions (for example, establishing a program on refusing to do dangerous tasks), and they also have effects that are difficult to measure, which are likely only to manifest themselves in the medium and long term. Thus, these results confirm the need for investing in training in the NTSs, since workers will continue experiencing difficulties, often unnecessary ones if the company had had more resources and a greater commitment to $\mathrm{OSH}$, for an indeterminate period of time.

Figure 2

Examples of actions of re-designing the socio-technical system which could reduce or eliminate the need for NTSs

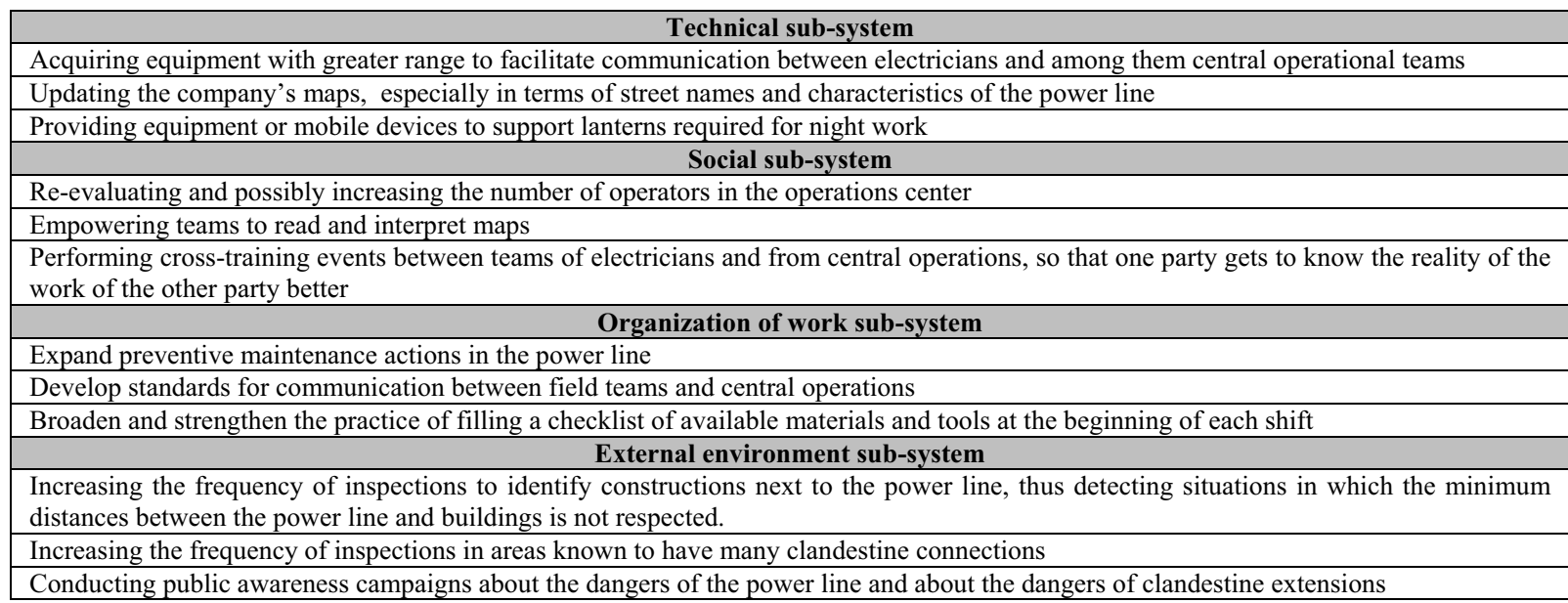

\section{Conclusions and discussion}

4.1. Operationalising the RE perspective when
identifying the NTSs

The main theoretical contribution of this article is to put forward recommendations for operationalizing the RE perspective when identifying NTS. Although the methods used for identifying NTSs are the same as those used in previous studies on NTS, the interpretation of the data obtained was the distinguishing factor. Thus, three actions can be highlighted to analyse data derived from the RE perspective: (a) identifying actions to re-design the sociotechnical system that can reduce or eliminate the need to use NTSs. These actions, together with the factors that can be incorporated into training scenarios, make clear that the conduct of NTSs is heavily dependent on the context in which they are conducted. Thus identifying the NTSs prompts discussion of the whole socio-technical system, thus making it clear that resilience is not only in individuals;

(b) classifying the NTSs into pragmatic categories that make sense to the experts in that field, and not paying heed to the categories traditionally used in previous studies (e.g, situational awareness), bearing in mind that this could imply the implicit adoption of epistemological foundations that conflict with those 
of RE. Thus, this decision has impacts that go beyond the mere establishment of labels for a given group of data;

(c) using the identification of the NTSs as a means of explaining the adaptations of the front-line operators, a topic of great interest to RE, to the extent that adaptations are one of the most visible manifestations of resilience. The RE perspective forces discussion of adaptations, which, in previous studies, would be neglected or else it would be implicitly assumed that the adaptations are undesirable and should not be covered in formal training programs.

\subsection{Useful NTSs for emergency maintenance teams}

A more practical contribution of this study, although it is also original for want of previous studies, concerns the identification of the 12 categories and 105 examples of NTS which are useful for electricity workers who work on emergency maintenance tasks on overhead distribution power lines.

However, it should be recognized that the NTS identified are not exhaustive and may not be fully applicable to other electricity distributors, which have striking differences in their socio-technical system. On the other hand, the significant effort in data collection and triangulation, as well as the participation of experts in the field in validating the results indicates that the NTSs are possibly of interest to other companies.

\subsection{Utility of the data collection methods}

The wealth of data generated by the analysis of cognitive tasks was also evident throughout the study. From the same database, it was possible to obtain three important results for the project: the NTSs, the factors that constitute training scenarios and the actions to re-design of the system. However, the contribution of the interviews, using the Critical Decisions Method, was the highlight. All 105 NTS samples came from interviews, and sometimes reinforced by other data sources.

In fact, this article opens up opportunities to continue in this direction, namely: (a) identifying NTS for control center operators, since they interact strongly with the teams that undertake emergency maintenance, (b) identifying NTSs for other services of an electricity distributor, such as mounting power lines, inspections and disconnections, (c) identifying
NTSs in other companies of the sector, in order to verify which are the most generalizable NTSs, and what the factors of the socio-technical problems are that often create more difficulties when these NTSs are conducted.

\subsection{Future studies}

The continuity of this work consists of developing the other stages of a training program in NTSs from the RE perspective, also covering the control center operators. In particular, emphasis will be given to designing training scenarios and forms of assessment, both of the performance of the workers and the training method.

It is also worth highlighting a need for a broader study aimed at establishing a robust theoretical basis for training in NTS under the RE perspective, and emphasizing the development of concepts, principles, methods and analysis of empirical results over the entire cycle of such training. Given the implications of RE, what should also be questioned is whether the expression, NTS, itself should be eliminated in this new theoretical framework with a view to drawing boundaries between the different perspectives.

\section{References}

[1] A. Mishra, K. Catchpole and P. McCulloch, The Oxford Nontechs system: reliability and validity of a tool for measuring teamwork behavior in the operating theatre, Qual Saf Health Care 18 (2009), 104-108.

[2] E. Hollnagel and D. Woods, Joint Cognitive Systems: foundations of cognitive systems engineering, Boca Raton: Taylor \& Francis / CRC, 2005.

[3] E. Hollnagel, Prologue: the scope of resilience engineering. In: E. Hollnagel, J. Pariès, D. Woods and J. Wreathall (Eds.), Resilience Engineering in Practice: a guidebook, Burlington: Ashgate, 2011.

[4] G. Fletcher, R. Flin, P. McGeorge, R. Glavin, N. Maran and R. Patey, Rating non-technical skills: developing a behavioral marker system for use in anaesthesia, Cognition, Technology and Work 6 (2004), 165-171.

[5] G. Klein, Macrocognitive measures for evaluating cognitive work. In: E. PATTERSON and J. MILLER, J. (Eds.), Macrocognition Metrics and Scenario: design and evaluation for real-world teams, Burlington: Ashgate, 2010.

[6] H. W. Hendrick and B. M. Kleiner, Macroergonomics: an introduction to work system design, Santa Monica: Human Factors and Ergonomics Society, 2001.

[7] J. BEergstrom, E. HEernriqson and N. Dahlstrom, From crew resource management to operational resilience, Proceedings of the fourth Resilience Engineering Symposium (2011), 36-42.

[8] L. A. Melo, G. Lima, N. Gomes and R. Soares, Segurança em serviços emergenciais em redes elétricas: os fatores ambientais, Revista Produção 13 (2003),88-101. 
[9] L. Gonçalves, Projeto, Implantação e Avaliação de Sistemas de Relatos de Incidentes: um estudo empírico em uma distribuidora de energia elétrica, Dissertação (Mestrado em Engenharia de Produção), UFRGS, 2011.

[10]M. T. Crichton and R. Flin, Identifying and training nontechnical skills of nuclear emergency response teams, Annals of Nuclear Energy 31 (2004), 1317-1330.

[11]N. Svedalis, S. Undre, J. Henry, E. Sydney, M. Koutantji, A. Darzi and C. Vincent, Development, initial reliability and validity testing of an observational tool for assessing technica skills of operating room nurses, International Journal of Nursing Studies 46 (2009), 1187-1193.

[12]R. Amalberti, The paradoxes of almost totally safe transportation systems, Safety Science 37 (2001), 109-126.
[13]R. Flin, P. O'Connor and M. Crichton, Safety at the Sharp End: a guide to non-technical skills, Burlington: Ashgate, 2008.

[14]S. Dekker and E. Hollnagel, Human factors and folk models, Cognition, Technology and Work 6 (2004), 79-86.

[15]S. Dekker, Drift into Failure: from hunting broken components to understanding complex systems, London: Ashgate, 2011.

[16]S. Dekker, The Field Guide to Understanding Human Error, London: Ashgate, 2006.

[17]T. A. Saurin and G. Carim Junior, Evaluation and improvement of a method for assessing HSMS from the resilience engineering perspective: a case study of an electricity distributor, Safety Science 49 (2011), 355-368 